\title{
Análisis categorial de cinco enfoques de la Didáctica de las Ciencias Experimentales a partir de la didáctica crítico- constructiva propuesta por Klafki
}

Henao Giraldo, Wilman Ricardo'

Categoría 2. Trabajo de investigación

\section{Resumen}

En esta investigación analizamos cinco enfoques de la didáctica de las ciencias experimentales a partir de la didáctica crítico-constructiva propuesta por Klafki: el aprendizaje por argumentación, el aprendizaje significativo crítico, el aprendizaje por resolución de problemas, enfoque CTS y asuntos socio-científicos. Para ello se planteó una investigación de corte cualitativo, hermenéutico- documental, tomando como categorías "la crítica inmanente, la crítica ideológica y la crítica terapéutica".

Dentro de los resultados de investigación se plantea la necesidad de teorizar al interior de los enfoques de la didáctica de las ciencias experimentales a partir de la teoría crítica terapéutica o crítica del sujeto.

\section{Palabras clave}

Didáctica de las ciencias experimentales, didáctica crítica, crítica inmanente, crítica ideológica, crítica terapéutica.

\section{Objetivos}

Se describió el campo de la didáctica de las ciencias experimentales, haciendo alusión a la construcción histórica que ha tenido la teoría, los principales enfoques y las producciones que se han dado en el campo. Posteriormente se analizaron los enfoques propuestos bajo las categorías críticas inmanente, ideológica y terapéutica.

1 Candidato a Doctor en Educación, Profesor Facultad de Educación Universidad de Antioquia - I. E Fe y Alegría Luis Amigó. whenaoo@yahoo.es. 
Revista Tecné, Episteme y Didaxis: TED. Año 2014, Número Extraordinario. ISSN Impreso: 0121-3814, ISSN web: 2323-0126

Memorias, Sexto Congreso Internacional sobre Formación de Profesores de Ciencias. 08 al 10 de octubre de 2014, Bogotá

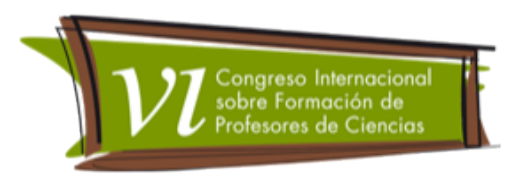

\section{Marco teórico}

Se retoma los postulados de la didáctica crítico-constructiva propuesta por Klafki, que se fundamenta en la teoría de la formación no afirmativa, y propone categorías para la selección de contenidos. Bajo la postura de Klafki se entiende la didáctica como campo conceptual y disciplinar que se encarga de problematizar y vigilar los procesos de enseñanza con respecto a la selección de contenidos, siendo su finalidad el entender cómo se lleva a cabo la formabilidad en el individuo.

\section{La crítica ideológica}

Aparece como el juicio histórico que se realiza a los contextos políticos e históricos, en los cuales los sujetos desarrollan sus vidas. La crítica ideológica, tiene la intensión de desmantelar los tejidos de poder para, desde una postura socioconstructivista, transformar las realidades históricas. Con ello, la crítica no se queda sólo en la denuncia desesperanzada, sino que abandera dinámicas de producción de nuevas realidades (Ospina, H.F., Alvarado, S.V. y Muñoz, D.A., 2008).

\section{La crítica terapéutica}

Pretende confrontar el proyecto vital de cada sujeto, a la luz de la responsabilidad que cada uno tiene con su propia vida. Esta crítica recupera los cimientos de las teorías de la formación, en cuanto reclama la función protagónica del sujeto en el proceso de darse forma. Lo terapéutico es entendido como la agencia del sujeto sobre sí mismo; el objetivo de la crítica se concentra en la problematización de los mundos de la vida y la conquista política. Se dirige hacia la autonomía y autorreflexividad del sujeto (Ibíd).

\section{La crítica inmanente}

Es entendida desde la perspectiva de Jürgen Habermas (1992:274), como la pretensión de recuperar los saberes sociales existentes en los trasfondos sociohistóricos de los mundos de la vida. La idea central de la crítica inmanente es la configuración de conocimientos teóricos que permitan trascender, sin desconocer las experiencias concretas de cada sujeto en sus relaciones cotidianas, los cuales operan como estrategias teóricas para el incremento de la reflexividad en dichos mundos de la vida. 
Revista Tecné, Episteme y Didaxis: TED. Año 2014, Número Extraordinario. ISSN Impreso: 0121-3814, ISSN web: 2323-0126

Memorias, Sexto Congreso Internacional sobre Formación de Profesores de Ciencias. 08 al 10 de octubre de 2014, Bogotá

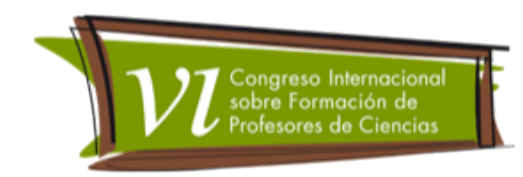

\section{Acercamiento al campo de la didáctica de las ciencias experimentales}

La evolución del status de la didáctica de las Ciencias, tiene que ver con la etapa de disciplina consolidada, propuesta por Adúriz-Bravo e Izquierdo (2002), en tanto que se relaciona con la creciente investigación en torno a la enseñanza y el aprendizaje de las ciencias, lo que se ve reflejado en el incremento de revisiones y resúmenes publicados al respecto. En los últimos años, la didáctica de las ciencias se ha ido conformando como dominio específico de conocimiento, con los elementos propios de una disciplina científica: una comunidad científica, unos órganos de expresión, unas líneas de investigación definidas y una evolución hacia consensos generalizados y hacia la integración de los distintos aspectos en cuerpos coherentes de conocimiento (Carrascosa, J., Gil, D. \& Martínez, F. ,1999: 22).

Analizando la correlación de la didáctica de las ciencias con otras disciplinas, podemos encontrar una estrecha relación con la epistemología de las ciencias, la historia de las ciencias, la psicología cognitiva; con disciplinas sociales como la lingüística, la sociología y la antropología que han provisto a la didáctica de las ciencias de fundamentos teóricos (Ibíd., pág. 69). Además, la didáctica de las ciencias permanece en un constante diálogo con la pedagogía, lo que les contribuye una retroalimentación bidireccional.

\section{Metodología}

\section{Paradigma o Enfoque}

El paradigma en el cual se enmarca el trabajo de investigación, es hermenéuticodocumental, que surge como respuesta a las tradiciones positivista e interpretativa y pretenden superar el reduccionismo de la primera y el conservadurismo de la segunda, admitiendo la posibilidad de una ciencia social que no sea ni puramente empírica ni sólo interpretativa (Foster, 1980, Citado por La Torre, Del Rincon, \&Torrasa, 1996).

El paradigma crítico introduce la ideología de forma explícita y la autorreflexión crítica en los procesos de conocimiento. Sus principios ideológicos tienen como finalidad la transformación de la estructura de las relaciones sociales, que se apoyan en la escuela de Frankfurt, en el neomarxisismo y en la teoría crítica social. 
Revista Tecné, Episteme y Didaxis: TED. Año 2014, Número Extraordinario. ISSN Impreso: 0121-3814, ISSN web: 2323-0126

Memorias, Sexto Congreso Internacional sobre Formación de Profesores de Ciencias. 08 al 10 de octubre de 2014, Bogotá

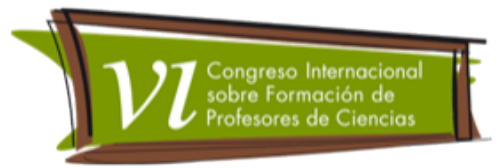

La perspectiva crítica tiene como objetivo el análisis de las transformaciones sociales y dar respuesta a determinados problemas generados por éstas. Algunos de sus principios son: a) Conocer y comprender la realidad como praxis b) Unir la teoría y la práctica; conocimiento, acción y valores. c) orientar el conocimiento hacia la emancipación y libertad del hombre, y d) implicar al docente a partir de la autorreflexión.

Desde este paradigma se cuestiona la supuesta neutralidad de la ciencia, y por ende de la investigación, a la que atribuye un carácter emancipativo y transformador de las organizaciones y procesos educativos. El grupo asume la responsabilidad de la investigación y propicia la reflexión, criticando los intereses, interrelaciones y prácticas educativas.

En las dimensiones conceptual y metodológica existen similitudes con el paradigma interpretativo, al que añade un componente ideológico con el fin de transformar la realidad además de describirla y comprenderla.

El enfoque en particular que se utiliza en la presente investigación es el hermenéutico - documental o reconstructiva, según Muñoz (2009) este enfoque es entendido desde la perspectiva de Jurgen Habermas como la pretensión de recuperar los saberes sociales existentes en los trasfondos socio-históricos de los mundos de la vida. La idea central de dicha hermenéutica reconstructiva es, de un lado, la configuración de conocimientos teóricos que permitan trascender sin desconocer las experiencias concretas de cada sujeto en sus relaciones cotidianas, las cuales operan como estrategias teóricas para el incremento de la reflexividad en dichos mundos de la vida. Por otra parte, "reconstrucción significa que se descompone una teoría y se la vuelve a recomponer en una forma nueva, con la finalidad de conseguir mejor el fin que ella se había propuesto." (Habermas, 1992 citado por Muñoz, 2009, pág. 274).

En dicho enfoque, se interpretan textos que convergen entre sí para dar determinada explicación de acuerdo a la construcción epistemológica de cada teoría, reflexionando sobre los puntos que se encuentran en tensión, si bien lo que propone la metodología es la reconstrucción de los textos frente a una propuesta dada, el cometido central de la reconstrucción en este autor es la emancipación del sujeto a través de la lucha por la racionalización de los mundosde la vida desde ejercicios de reflexión y argumentación, y con ello, el rechazo de su colonización por parte de los imperativos funcionales del sistema (Muñoz, 2009) 
Revista Tecné, Episteme y Didaxis: TED. Año 2014, Número Extraordinario. ISSN Impreso: 0121-3814, ISSN web: 2323-0126

Memorias, Sexto Congreso Internacional sobre Formación de Profesores de Ciencias. 08 al 10 de octubre de 2014, Bogotá

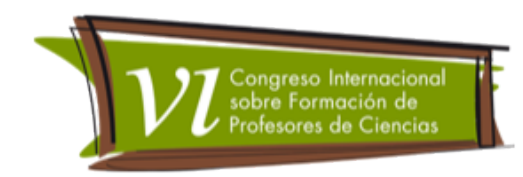

\section{Proceso de Recolección de la información}

Esta investigación se ubica en el marco de investigación documental y tiene como objeto de estudio analizar las siguientes categorías:

a. La crítica inmanente.

b. La crítica ideológica.

c. La crítica ideológica.

A la luz de estas categorías se revisaron cinco enfoques de la didáctica de las ciencias experimentales a partir de la didáctica crítico-constructiva propuesta por Klafki: el aprendizaje por argumentación, el aprendizaje significativo crítico, el aprendizaje por resolución de problemas, enfoque CTS y asuntos socio-científicos.

Se utilizara como técnica el análisis de contenido, al ofrecer la posibilidad de investigar sobre la naturaleza del discurso, utilizando como instrumentos de compendio de datos de los documentos. Este tipo de trabajos genera condiciones de posibilidad para que otros investigadores de este campo teórico se pronuncien sobre él mismo, tejiendo nuevas elaboraciones conducentes a ampliar, delimitar, comparar, algunos aspectos que dan cuenta de la criticidad de los enfoque en la didáctica de las ciencias.

\section{Resultados}

A continuación se presentan los análisis de cada una de las categorías propuestas:

\section{Análisis Crítico al enfoque en CTS}

La didáctica de las ciencias, retoma el enfoque CTS para configurar, desde la educación, aspectos como la alfabetización científica y tecnológica de la ciudadanía.

En la siguiente tabla se presenta el análisis del enfoque CTS (ver tabla 1). 
Revista Tecné, Episteme y Didaxis: TED. Año 2014, Número Extraordinario. ISSN Impreso: 0121-3814, ISSN web: 2323-0126

Memorias, Sexto Congreso Internacional sobre Formación de Profesores de Ciencias. 08 al 10 de octubre de 2014, Bogotá

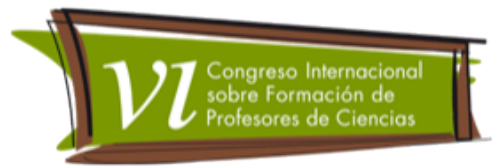

Tabla 1. Criticidad del enfoque CTS.

\begin{tabular}{|c|l|}
\hline Categorías & \multicolumn{1}{|c|}{ Enfoque CTS } \\
\hline Inmanente & $\begin{array}{l}\text { Su campo específico es problematizar } \\
\text { como se toman los asuntos científicos para } \\
\text { ser llevados a la sociedad. }\end{array}$ \\
\hline Ideológica & $\begin{array}{l}\text { Hace explícita las tensiones (políticas, } \\
\text { relaciones de poder) producidas al } \\
\text { momento de la construcción del } \\
\text { conocimiento científico. }\end{array}$ \\
\hline Sujeto & \begin{tabular}{l} 
No se evidencia. \\
\hline
\end{tabular} \\
\hline
\end{tabular}

\section{Análisis crítico de la teoría de asuntos socio-científicos}

Esta teoría permite la reflexión del sujeto hacia sí mismo; es decir, saber cómo la nueva información a la que se está enfrentando puede afectarlo y cuál son sus posturas frente a estos.

Se puede decir que este enfoque, presenta las tres categorías propuestas para una perspectiva crítico-constructiva (ver tabla 2).

Tabla 2. Criticidad del enfoque asuntos socio-científicos.

\begin{tabular}{|c|l|}
\hline Categorías & \multicolumn{1}{|c|}{ Enfoque de los asuntos socio-científicos } \\
\hline Inmanente & $\begin{array}{l}\text { Su campo específico se interesa por la } \\
\text { conformación de los argumentos y los tipos de } \\
\text { análisis que los estudiantes hacen frente a un tema } \\
\text { relacionado con la ciencia que se encuentra en } \\
\text { plena discusión. }\end{array}$ \\
\hline Ideológica & $\begin{array}{l}\text { Es clave que la discusión que se teje, de cuenta de } \\
\text { los aspectos económicos, la influencia de las } \\
\text { políticas en emergencia y de la cuestión moral de } \\
\text { los asuntos socio- científicos que se están tratando. }\end{array}$ \\
\hline
\end{tabular}


Revista Tecné, Episteme y Didaxis: TED. Año 2014, Número Extraordinario. ISSN Impreso: 0121-3814, ISSN web: 2323-0126

Memorias, Sexto Congreso Internacional sobre Formación de Profesores de Ciencias. 08 al 10 de octubre de 2014, Bogotá

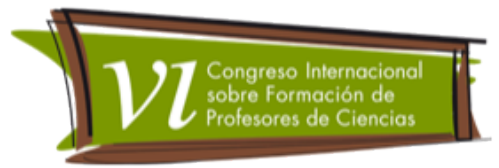

\begin{tabular}{|l|l|}
\hline \hline \multicolumn{1}{|c|}{} & $\begin{array}{l}\text { En estudios realizados por Zeidler, D. L., Sadler, T. D., } \\
\text { Simmons, M. L., \& Howes, E. V. (2005), toman los } \\
\text { asuntos socio-científicos para evidenciar los tipos de } \\
\text { razonamiento de los estudiantes y los tipos de } \\
\text { decisiones que toman frente a determinada } \\
\text { situación. Se muestra además, la influencia que se } \\
\text { tiene de las experiencias personales, las } \\
\text { consideraciones emotivas frente a las situaciones } \\
\text { planteadas. }\end{array}$ \\
\hline
\end{tabular}

Se puede afirmar que esta es una de las líneas de investigación en educación en ciencias, que poseen mayor consistencia al tener el sentido crítico, pues se evidencia como cumple con las tres categorías de análisis retomadas por Klafki para abordar el sentido crítico-constructivo.

\section{Análisis crítico de la Teoría aprendizaje por argumentación}

En el aprendizaje por argumentación, se encontró una ausencia de la categoría "sujeto". Esto significa que no se encuentra la posibilidad de que el sujeto realice un análisis de sí mismo con relación a la teoría científica, concluyendo que bajo los criterios planteados por Klafki, el aprendizaje por argumentación no se entendería como una perspectiva crítica (ver tabla 3).

Tabla 3. Criticidad del enfoque aprendizaje por argumentación.

\begin{tabular}{|c|l|}
\hline Categorías & \multicolumn{1}{|c|}{ Enfoque aprendizaje por argumentación } \\
\hline Inmanente & $\begin{array}{l}\text { Es su interés la elaboración de argumentos, tiene en } \\
\text { cuenta los supuestos de la lingüística y la } \\
\text { comunicación. Toma las bases de la naturaleza de las } \\
\text { ciencias experimentales y su relación con la } \\
\text { alfabetización científica. }\end{array}$ \\
\hline Ideológica & $\begin{array}{l}\text { Aboga por el pensamiento crítico como base para el } \\
\text { desarrollo de una sociedad política y democrática. El } \\
\text { estudiante participa en el discurso social. }\end{array}$ \\
\hline Sujeto & No se evidencia. \\
\hline
\end{tabular}


Revista Tecné, Episteme y Didaxis: TED. Año 2014, Número Extraordinario. ISSN Impreso: 0121-3814, ISSN web: 2323-0126

Memorias, Sexto Congreso Internacional sobre Formación de Profesores de Ciencias. 08 al 10 de octubre de 2014, Bogotá

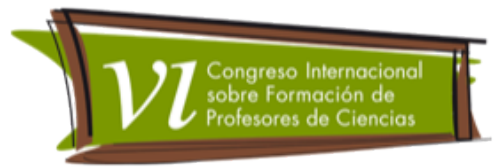

\section{Análisis crítico de la Teoría del aprendizaje significativo crítico}

Los esfuerzos de los investigadores por realizar una delimitación del campo y evidenciar trabajos fructíferos en la línea, ha hecho que tenga alta aplicabilidad en el aula (Rodriguez, M. L.; Moreira, M. A.; Caballero, M. C. \& Greca, I. M., 2008), se reconocen además, las contribuciones que generan los otros enfoques a la teoría.

La siguiente tabla muestra los resultados de la criticidad del enfoque aprendizaje significativo crítico (ver tabla 4).

Tabla 2. Criticidad del enfoque aprendizaje significativo crítico.

\begin{tabular}{|c|l|}
\hline Categorías & \multicolumn{1}{|c|}{ Enfoque aprendizaje significativo crítico } \\
\hline Inmanente & $\begin{array}{l}\text { El aprendizaje significativo crítico, es aquella } \\
\text { perspectiva que permite al sujeto, formar parte de su } \\
\text { cultura y al mismo tiempo estar fuera de ella. Se trata } \\
\text { de una perspectiva antropológica en relación con las } \\
\text { actividades de su grupo social, que permite al } \\
\text { individuo participar de tales actividades. } \\
\text { Rescata la interacción social y el proceso de } \\
\text { enseñanza y aprendizaje basado en preguntas más } \\
\text { que en ofrecer respuestas. }\end{array}$ \\
\hline Ideológica & No se evidencia \\
\hline Sujeto & No se evidencia. \\
\hline
\end{tabular}

\section{Análisis crítico de la teoría Resolución de problemas}

Se puede decir, que el enfoque de aprendizaje por resolución de problemas, presenta la definición de la crítica inmanente, pues caracteriza sus fronteras teóricas, evidenciando en que se diferencia de los otros enfoques (ver tabla 5). 
Revista Tecné, Episteme y Didaxis: TED. Año 2014, Número Extraordinario. ISSN Impreso: 0121-3814, ISSN web: 2323-0126

Memorias, Sexto Congreso Internacional sobre Formación de Profesores de Ciencias. 08 al 10 de octubre de 2014, Bogotá

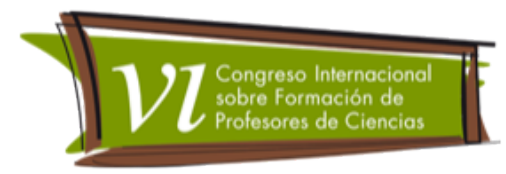

Tabla 5. Criticidad de la Teoría de resolución de problemas.

\begin{tabular}{|c|l|}
\hline Categorías & \multicolumn{1}{|c|}{ Teoría de la resolución de problemas } \\
\hline Inmanente & $\begin{array}{l}\text { El enfoque por resolución de problemas de la } \\
\text { didáctica de las ciencias, busca el mejoramiento de } \\
\text { varios aspectos esenciales de la cultura, como lo son } \\
\text { el desarrollo social y el aprendizaje de la cultura por } \\
\text { parte de los individuos, a través de la construcción y } \\
\text { desarrollo de situaciones problema. }\end{array}$ \\
\hline Ideológica & No se evidencia \\
\hline Sujeto & No se evidencia. \\
\hline
\end{tabular}

\section{Conclusiones}

En general, todos los enfoques mostraron un estrecho vínculo con la psicología cognitiva y por ende una alta preocupación por el aprendizaje de los conceptos científicos, cuestión que se ve reflejada en la categoría crítica inmanente, donde a pesar de los matices, esa es la preocupación de todos los enfoques. Esto podría deberse a que la psicología cognitiva ha sido una de las ciencias donde la didáctica de las ciencias experimentales ha encontrado piso teórico para comprender el proceso de adquisición del conocimiento, para nuestro caso, el científico.

Es de rescatar que los enfoques CTS, asuntos socio-científicos, aprendizaje significativo crítico y aprendizaje por argumentación, evidencian una crítica ideológica; con ello se muestra, que si bien, las perspectivas críticas están dadas desde una perspectiva critico-constructiva y si bien, no son la base para el desarrollo de los enfoques a nivel teórico, se nota esfuerzo por llevar una ciencia a las aulas que se encuentra en construcción y la necesidad de mostrar las tensiones y las relaciones de poder al momento de producir la ciencia. También se evidencia el esfuerzo por demostrar cómo influyen los asuntos sociales y políticos cuando se produce conocimiento científico. 
Revista Tecné, Episteme y Didaxis: TED. Año 2014, Número Extraordinario. ISSN Impreso: 0121-3814, ISSN web: 2323-0126

Memorias, Sexto Congreso Internacional sobre Formación de Profesores de Ciencias. 08 al 10 de octubre de 2014, Bogotá

Este trabajo deja abiertas muchos aspectos para futuras investigaciones: la teorización de las categorías faltantes en cada uno de los enfoques y, el diseño de estrategias que se puedan validar en la cotidianidad y en el contexto escolar.

\section{Referencias bibliográficas}

Adúriz-Bravo, A. \& Izquierdo, M. (2002). Acerca de la didáctica de las ciencias como disciplina autónoma. Revista Electrónica de Enseñanza de las ciencias, 1 (3), $130 \quad-\quad 140 . \quad$ Recuperado de http://reec.uvigo.es/volumenes/volumen1/Numero3/Art1.pdf

Carrascosa, J., Gil, D. \& Martínez, F. (1999). El surgimiento de la Didáctica de las Ciencias como campo específico de conocimientos. Educación y Pedagogía, XI (25), $15-65$.

Habermas, J. (1992). La reconstrucción del materialismo histórico. Madrid: Taurus Humanidades.

Ospina, H.F., Alvarado, S.V. y Muñoz, D.A. (2008). La educación como transformación y la formación como emancipación: apuntes conceptuales desde la ciencia crítica de la educación. Revista Alternativas. Serie Historia y Prácticas Pedagógicas, 1 - 15.

Rodriguez, M. L.; Moreira, M. A.; Caballero, M. C. \& Greca, I. M. (2008). La teoría del Aprendizaje Significativo en la Perspectiva de la Psicología Cognitiva. Barcelona: Octaedro SL.

Zeidler, D. L., Sadler, T. D., Simmons, M. L., \& Howes, E. V. (2005). Beyond STS: Aresearch-based framework for socioscientific issues education. Science Education, 89 (3), 357-377. 